\title{
STATISTICAL LIMIT SUPERIOR AND LIMIT INFERIOR
}

\author{
J. A. FRIDY AND C. ORHAN
}

(Communicated by J. Marshall Ash)

\begin{abstract}
Following the concept of statistical convergence and statistical cluster points of a sequence $x$, we give a definition of statistical limit superior and inferior which yields natural relationships among these ideas: e.g., $x$ is statistically convergent if and only if st-liminf $x=\operatorname{st-limsup} x$. The statistical core of $x$ is also introduced, for which an analogue of Knopp's Core Theorem is proved. Also, it is proved that a bounded sequence that is $C_{1}$-summable to its statistical limit superior is statistically convergent.
\end{abstract}

\section{INTRODUCTION}

The concepts of limit and cluster point of a sequence $x$ have been extended ([7], [8] and [9]) to st-lim $x$ and statistical cluster point using the natural density $\delta$ of a set $K$ of positive integers:

$$
\left.\delta\{K\}:=\lim _{n} \frac{1}{n} \text { (the number } k \leq n \text { such that } k \in K\right) .
$$

(See [18], Chapter 11 for a basic theory of density.) The sequence $x$ is statistically convergent to $L$, denoted $\operatorname{st-lim} x=L$, if for every $\epsilon>0, \delta\left\{k:\left|x_{k}-L\right| \geq \epsilon\right\}=0$. Over the years, statistical convergence has been examined in number theory [6], trigonometric series [19] and summability theory [10]. It has also been considered in locally convex spaces [16]. Recently, generalizations of statistical convergence have been made in the study of strong integral summability [3] and the structure of ideals of bounded continuous functions on locally compact spaces [4]. Statistical convergence and its generalizations are also connected with subsets of the StoneCech compactification of the natural numbers [5]. A matrix characterization and a measure theoretical subsequence characterization of statistical convergence may be found in [11] and [17], respectively.

The number $\gamma$ is called a statistical cluster point of $x$ if for every $\epsilon>0$ the set $\left\{k:\left|x_{k}-\gamma\right|<\epsilon\right\}$ does not have density zero. The purpose this paper is to present natural definitions of the concepts of statistical limit superior and inferior and to develop some statistical analogues of properties of the ordinary limit superior and inferior. The latter results include statistical analogues of Knopp's Core Theorem [13] and R.C. Buck's Theorem [1] on Cesàro summability of a sequence to its limit superior.

Received by the editors April 20, 1995 and, in revised form, July 15, 1996

1991 Mathematics Subject Classification. Primary 40A05; Secondary 26A03, 11B05.

Key words and phrases. Natural density, statistically convergent sequence, statistical cluster point, core of a sequence.

(C) 1997 American Mathematical Society 


\section{Definitions AND BASIC RESUlts}

Throughout the paper $k$ and $n$ will always denote positive integers; $x, y$, and $z$ will denote real number sequences; and $\mathbb{N}$ and $\mathbb{R}$ will denote the sets of positive integers and real numbers, respectively. If $K \subseteq \mathbb{N}$, then $K_{n}:=\{k: k \leq n\}$, and $\left|K_{n}\right|$ denotes the cardinality of $K_{n}$.

For a real number sequence $x$ let $B_{x}$ denote the set:

$$
B_{x}:=\left\{b \in \mathbb{R}: \delta\left\{k: x_{k}>b\right\} \neq 0\right\} ;
$$

similarly,

$$
A_{x}:=\left\{a \in \mathbb{R}: \delta\left\{k: x_{k}<a\right\} \neq 0\right\} .
$$

Note that throughout this paper the statement $\delta\{K\} \neq 0$ means that either $\delta\{K\}>$ 0 or $K$ does not have natural density.

Definition 1. If $x$ is a real number sequence, then the statistical limit superior of $x$ is given by

$$
\text { st-lim } \sup x:=\left\{\begin{array}{lll}
\sup B_{x}, & \text { if } & B_{x} \neq \emptyset, \\
-\infty & \text { if } & B_{x}=\emptyset .
\end{array}\right.
$$

Also, the statistical limit inferior of $x$ is given by

$$
\text { st-lim } \inf x:= \begin{cases}\inf A_{x}, & \text { if } \quad A_{x} \neq \emptyset, \\ +\infty, & \text { if } \quad A_{x}=\emptyset .\end{cases}
$$

A simple example will help to illustrate the concepts just defined. Let the sequence $x$ be given by

$$
x_{k}:=\left\{\begin{array}{llll}
k, & \text { if } & k & \text { is an odd square, } \\
2, & \text { if } & k & \text { is an even square } \\
1, & \text { if } & k & \text { is an odd nonsquare, } \\
0, & \text { if } & k & \text { is an even nonsquare. }
\end{array}\right.
$$

Note that although $x$ is unbounded above, it is "statistically bounded" because the set of squares has density zero. Thus $B_{x}=(-\infty, 1)$ and st-lim $\sup x=1$. Also, $x$ is not statistically convergent since it has two (disjoint) subsequences of positive density that converge to 0 and 1 , respectively. (See Theorem 1 of [8].) Also note that the set of statistical cluster points of $x$ is $\{0,1\}$, and st-lim $\sup x$ equals the greatest element while st-lim inf is the least element of this set. This observation suggests the main idea of the first theorem, which can be proved by a straightforward least upper bound argument.

Theorem 1. If $\beta=\operatorname{st}-\lim \sup x$ is finite, then for every positive number $\epsilon$

$$
\delta\left\{k: x_{k}>\beta-\epsilon\right\} \neq 0 \quad \text { and } \quad \delta\left\{k: x_{k}>\beta+\epsilon\right\}=0 .
$$

Conversely, if (1) holds for every positive $\epsilon$ then $\beta=\operatorname{st-lim} \sup x$.

The dual statement for st-lim inf $x$ is as follows.

Theorem $\mathbf{1}^{\prime}$. If $\alpha=\operatorname{st-liminf} x$ is finite, then for every positive number $\epsilon$

$$
\delta\left\{k: x_{k}<\alpha+\epsilon\right\} \neq 0 \quad \text { and } \quad \delta\left\{k: x_{k}<\alpha-\epsilon\right\}=0 .
$$

Conversely, if (2) holds for every positive $\epsilon$ then $\alpha=\operatorname{st}-\liminf x$. 
From the definition of statistical cluster point in [9] we see that Theorems 1 and $1^{\prime}$ can be interpreted as saying that st- $\lim \sup x$ and st- $\lim \inf x$ are the greatest and least statistical cluster points of $x$. The next theorem reinforces that observation.

Theorem 2. For any sequence $x$, st- $\liminf x \leq \operatorname{st-lim\operatorname {sup}x}$.

Proof. First consider the case in which st- $\lim \sup x=-\infty$. This implies that $B_{x}=\emptyset$, so for every $b$ in $\mathbb{R}, \delta\left\{k: x_{k}>b\right\}=0$. This implies that $\delta\left\{k: x_{k} \leq b\right\}=1$, so for every $a$ in $\mathbb{R}, \delta\left\{k: x_{k}<a\right\} \neq 0$. Hence, st- $\lim \inf x=-\infty$.

The case in which st- $\lim \sup x=+\infty$ needs no proof, so we next assume that $\beta=\operatorname{st}-\lim \sup x$ is finite, and let $\alpha:=\operatorname{st}-\liminf x$. Given $\epsilon>0$ we show that $\beta+\epsilon \in A_{x}$, so that $\alpha \leq \beta+\epsilon$. By Theorem 1, $\delta\left\{k: x_{k}>\beta+\epsilon / 2\right\}=0$ because $\beta=$ lub $B_{x}$. This implies that $\delta\left\{k: x_{k} \leq \beta+\frac{\epsilon}{2}\right\}=1$ which, in turn, implies that $\delta\left\{k: x_{k}<\beta+\epsilon\right\}=1$. Hence, $\beta+\epsilon \in A_{x}$. By definition $\alpha=\inf A_{x}$, so we conclude that $\alpha \leq \beta+\epsilon$; and since $\epsilon$ is arbitrary this gives us $\alpha \leq \beta$.

From Theorem 2 and the above definition, it is clear that

$$
\liminf x \leq \operatorname{st}-\liminf x \leq \operatorname{st}-\lim \sup x \leq \lim \sup x
$$

for any sequence $x$.

A statistical limit point of a sequence $x$ is defined in [9] as the limit of a subsequence of $x$ whose indices do not have zero density. Since it was noted there that a bounded sequence might have no statistical limit point, one cannot say that st- $\lim \sup x$ is equal to the greatest such point. (Compare this to the remark following Theorem 1.) See, for example, Example 4 of [9]. This suggests the following question:

If $x$ does have a greatest statistical limit point $\mu$, does it follow that $\mu=$ st- $\lim \sup x$ ?

The answer is "no," which is shown by the following sequence.

Example 1. Following Example 4 of [9] we let $u$ be the uniformly distributed sequence $u=\left\{0,1,0, \frac{1}{2}, 1,0, \frac{1}{3}, \frac{2}{3}, 1,0, \frac{1}{4}, \frac{2}{4}, \frac{3}{4}, 1, \ldots\right\}$, and define

$$
x_{2 k-1}:=0 \text { and } x_{2 k}:=u_{k} .
$$

Then st- $\lim \sup x=1$ because $\delta\left\{k: x_{k}>1-\epsilon\right\}=\epsilon / 2$. Also, zero is the only statistical limit point of $x$ because $u$ has none (as shown in [9]). Hence, the greatest statistical limit point of $x$ is zero, but st- $\lim \sup x=1$.

The next result is another statistical analogue of a very basic property of convergent sequences. For clarity of presentation we first give a formal definition of another statistical concept.

Definition 2. The real number sequence $x$ is said to be statistically bounded if there is a number $B$ such that $\delta\left\{k:\left|x_{k}\right|>B\right\}=0$.

Note that statistical boundedness implies that st- lim sup and st- lim inf are finite, so Properties (1) and (2) of Theorems 1 and $1^{\prime}$ hold.

Theorem 3. The statistically bounded sequence $x$ is statistically convergent if and only if

$$
\text { st- } \liminf x=\text { st- } \lim \sup x \text {. }
$$


Proof. Let $\alpha:=\operatorname{st}-\liminf x$ and $\beta:=\operatorname{st-} \lim \sup x$. First assume that st- $\lim x=L$ and $\epsilon>0$. Then $\delta\left\{k:\left|x_{k}-L\right| \geq \epsilon\right\}=0$, so $\delta\left\{k: x_{k}>L+\epsilon\right\}=0$, which implies that $\beta \leq L$. We also have $\delta\left\{k: x_{k}<L-\epsilon\right\}=0$ which implies that $L \leq \alpha$. Therefore $\beta \leq \alpha$, which we combine with Theorem 2 to conclude that $\alpha=\beta$.

Next assume $\alpha=\beta$ and define $L:=\alpha$. If $\epsilon>0$ then (1) and (2) of Theorems 1 and $1^{\prime}$ imply $\delta\left\{k: x_{k}>L+\frac{\epsilon}{2}\right\}=0$ and $\delta\left\{k: x_{k}<L-\frac{\epsilon}{2}\right\}=0$. Hence, st- $\lim x=L$.

\section{Summability theOREMS CONCERNING ST - LIM SUP}

In [13] Knopp introduced the concept of the core of a sequence and proved the well-known Core Theorem. Since the core of a bounded sequence $x$ is the closed convex hull of the set of limit points of $x$, we can replace limit points with statistical cluster points to produce a natural analogue of Knopp's core.

Definition 3. If $x$ is a statistically bounded sequence, then the statistical core of $x$ is the closed interval $[\operatorname{st}-\lim \inf x, \operatorname{st}-\lim \sup x]$. In case $x$ is not statistically bounded, st-core $\{x\}$ is defined accordingly as either [st- $\lim \inf x, \infty),(-\infty, \infty)$, or $(-\infty, \operatorname{st}-\lim \sup x]$.

We shall denote the statistical core of $x$ by st-core $\{x\}$, and $K$-core $\{x\}$ will denote the usual core. It is clear from (3) that for any real sequence $x$

$$
\text { st-core }\{x\} \subseteq K \text {-core }\{x\} .
$$

Recall that the Core Theorem asserts that $K$-core $\{A x\} \subseteq K$-core $\{x\}$, whenever $A x$ exists for the nonnegative regular matrix $A[12$, p. 55]. In [15] Maddox proves a variant of the Core Theorem that $\lim \sup A x \leq \lim \sup x$ for every bounded $x$ if and only if $A$ is regular and $\lim _{n} \sum_{k=0}^{\infty}\left|a_{n k}\right|=1$. We shall prove a similar result for the st-core $\{x\}$. For this purpose let us recall some previous results and notations. In [2] Connor proved that the set of bounded statistically convergent sequences is equal to the set of bounded strongly $p$-Cesàro summable sequences $\left(S \sqcap \ell_{\infty}=\omega_{p} \sqcap \ell_{\infty}\right)$. In [14] Maddox proved that a matrix $A$ maps $\omega_{p} \sqcap \ell_{\infty}$ into $c$ if and only if $A$ is in the class $\mathcal{T}^{*}$, i.e., $A$ is regular and $\lim _{n} \sum_{k \in E}\left|a_{n k}\right|=0$ for every $E \subseteq \mathbb{N}$ such that $\delta\{E\}=0$. Throughout the following we shall use the abbreviations

$$
\alpha(x):=\operatorname{st}-\liminf x \text { and } \beta(x):=\operatorname{st}-\lim \sup x .
$$

Lemma. Suppose the matrix A satisfies $\sup _{n} \sum_{k=1}^{\infty}\left|a_{n k}\right|<\infty$; then

$$
\lim \sup A x \leq \mathrm{st}-\lim \sup x \quad \text { for every } x \in \ell_{\infty}
$$

if and only if

$$
A \in \mathcal{T}^{*} \quad \text { and } \quad \lim _{n} \sum_{k=1}^{\infty}\left|a_{n k}\right|=1 .
$$

Proof. Assume $A$ satisfies (4) and $x \in \ell_{\infty}$. Then $\beta(x) \leq \limsup x$ and, since $\sup _{n} \sum_{k}\left|a_{n k}\right|<\infty, A x \in \ell_{\infty}$. By (4) we have

$$
-\beta(-x) \leq-\lim \sup (-A x) \leq \lim \sup A x \leq \beta(x),
$$


or

$$
\text { st }-\liminf x \leq \liminf A x \leq \limsup A x \leq \beta(x)
$$

If $x \in S \sqcap \ell_{\infty}$ we have $\alpha(x)=\beta(x)=$ st - $\lim x$, so (6) implies that $\lim A x=$ st $-\lim x$. Hence, $A$ maps $S \sqcap \ell_{\infty}$ into $c$, so by the theorems of Maddox and Connor, $A \in \mathcal{T}^{*}$. Also, since $\beta(x) \leq \lim \sup x$, (4) implies that $\lim \sup A x \leq \lim \sup x$, and Maddox's variant of Knopp's Core Theorem yields

$$
\lim _{n} \sum_{k=1}^{\infty}\left|a_{n k}\right|=1
$$

Conversely, assume (5) and let $x$ be bounded; then $A x \in \ell_{\infty}$ and $\beta(x)$ is finite. Given $\epsilon>0$ let $E:=\left\{k: x_{k}>\beta(x)+\epsilon\right\}$. Thus $\delta\{E\}=0$, and if $k \notin E$ then $x_{k} \leq \beta(x)+\epsilon$. For any real number $z$ we write

$$
z^{+}:=\max \{z, 0\} \quad \text { and } \quad z^{-}:=\max \{-z, 0\},
$$

whence

$$
|z|=z^{+}+z^{-}, \quad z=z^{+}-z^{-}, \text {and }|z|-z=2 z^{-} .
$$

For a fixed positive integer $m$ we write

$$
\begin{aligned}
(A x)_{n} & =\sum_{k<m} a_{n k} x_{k}+\sum_{k \geq m} a_{n k} x_{k} \\
& =\sum_{k<m} a_{n k} x_{k}+\sum_{k \geq m} a_{n k}^{+} x_{k}-\sum_{k \geq m} a_{n k}^{-} x_{k} \\
\leq & \|x\|_{\infty} \sum_{k<m}\left|a_{n k}\right|+\sum_{\substack{k \geq m \\
k \notin E}} a_{n k}^{+} x_{k}+\sum_{\substack{k \geq m \\
k \in E}} a_{n k}^{+} x_{k}+\|x\|_{\infty} \sum_{k \geq m}\left(\left|a_{n k}\right|-a_{n k}\right) \\
\leq & +(\beta(x)+\epsilon) \sum_{\substack{k \geq m \\
k \notin E}}\left|a_{n k}\right|+\|x\|_{\infty} \sum_{\substack{k \geq m \\
k \in E}}\left|a_{n k}\right| \\
& +\|x\|_{\infty} \sum_{k \geq m}\left(\left|a_{n k}\right|-a_{n k}\right) .
\end{aligned}
$$

Taking the limit superior as $n \longrightarrow \infty$ and using (5) and the regularity of $A$, we get

$$
\lim \sup (A x)_{n} \leq \beta(x)+\epsilon .
$$

Since $\epsilon$ is arbitrary we conclude that (4) holds, and the proof is complete.

It is clear that one can prove a similar result for $\alpha(x) \leq \lim \inf A x$, and therefore we have the following result.

Theorem 4 (Statistical Core Theorem). If the matrix A satisfies $\sup _{n} \sum_{k=1}^{\infty}\left|a_{n k}\right|<$ $\infty$, then

$$
K-\operatorname{core}\{A x\} \subseteq \text { st-core }\{x\} \text { for every } x \text { in } \ell_{\infty}
$$


if and only if

$$
A \in \mathcal{T}^{*} \quad \text { and } \quad \lim _{n} \sum_{k=1}^{\infty}\left|a_{n k}\right|=1 .
$$

In [1] Buck proved that a sequence that is $C_{1}$-summable to its limit superior is statistically convergent. The next theorem is a statistical analogue of that result.

Theorem 5. If the sequence $x$ is bounded above and $C_{1}$-summable to the number $\beta:=\operatorname{st}-\lim \sup x$, then $x$ is statistically convergent to $\beta$.

Proof. Suppose that $x$ is not statistically convergent to $\beta$. Then by Theorem 3 , st- $\liminf x<\beta$, so there is a number $\mu<\beta$ such that $\delta\left\{k: x_{k}<\mu\right\} \neq 0$. Let $K^{\prime}:=\left\{k: x_{k}<\mu\right\}$. By the definition of $\beta, \delta\left\{k: x_{k}>\beta+\epsilon\right\}=0$ for every $\epsilon>0$. Define

$$
K^{\prime \prime}:=\left\{k: \mu \leq x_{k} \leq \beta+\epsilon\right\} \quad \text { and } \quad K^{\prime \prime \prime}:=\left\{k: x_{k}>\beta+\epsilon\right\},
$$

and let $B:=\sup _{k} x_{k}<\infty$. Since $\delta\left\{K^{\prime}\right\} \neq 0$, there are infinitely many $n$ such that

$$
\frac{1}{n}\left|K_{n}^{\prime}\right| \geq d>0
$$

and for each such $n$ we have

$$
\begin{aligned}
\left(C_{1} x\right)_{n} & =\frac{1}{n} \sum_{k \in K_{n}^{\prime}} x_{k}+\frac{1}{n} \sum_{k \in K_{n}^{\prime \prime}} x_{k}+\frac{1}{n} \sum_{k \in K_{n}^{\prime \prime \prime}} x_{k} \\
& <\frac{\mu}{n}\left|K_{n}^{\prime}\right|+\frac{\beta+\epsilon}{n}\left|K_{n}^{\prime \prime}\right|+\frac{B}{n}\left|K_{n}^{\prime \prime \prime}\right| \\
& =\mu \frac{\left|K_{n}^{\prime}\right|}{n}+(\beta+\epsilon)\left(1-\frac{\left|K_{n}^{\prime}\right|}{n}\right)+o(1) \\
& \leq \beta-d(\beta-\mu)+\epsilon(1-d)+o(1) .
\end{aligned}
$$

Since $\epsilon>0$ is arbitrary it follows that

$$
\liminf C_{1} x \leq \beta-d(\beta-\mu)<\beta .
$$

Hence, $x$ is not $C_{1}$-summable to $\beta$, which completes the proof.

By symmetry we have the dual result for lower bounds.

Corollary. If the sequence $x$ is bounded below and $C_{1}$-summable to the number $\alpha:=\operatorname{st}-\liminf x$, then $x$ is statistically convergent to $\alpha$.

Since Buck's Theorem, which was the motivation for Theorem 5, does not assume an upper bound of the sequence, it is natural to ask if that hypothesis could be eliminated from Theorem 5. The following example shows that the upper bound cannot be omitted or even replaced by the weaker assumption of a statistical upper bound.

Example 2. Let $x$ be the sequence given by

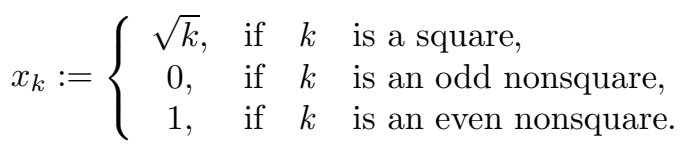

Since $\delta\left\{k: x_{k}=0\right\}=1 / 2=\delta\left\{k: x_{k}=1\right\}$, it is clear that st-liminf $x=0$ and st- $\lim \sup x=1$. Therefore $x$ is not statistically convergent. Also note that $x$ is 
statistically bounded since $\delta\left\{k:\left|x_{k}\right|>1\right\}=0$. It remains to show that $C_{1} x$ has limit $1=$ st - $\lim \sup x$. Let $K^{2}$ denote the set of squares, and let $K^{0}$ and $K^{1}$ denote, respectively, the sets of odd and even nonsquares. With $[t]:=\max \{k: k \leq t\}$, this yields

$$
\begin{aligned}
\left(C_{1} x\right)_{n} & =\frac{1}{n} \sum_{k \in K_{n}^{0}} x_{k}+\frac{1}{n} \sum_{k \in K_{n}^{1}} x_{k}+\frac{1}{n} \sum_{k \in K_{n}^{2}} x_{k} \\
& =0+\frac{1}{n} \frac{[n-[\sqrt{n}]]}{2}+\frac{1}{n} \sum_{i \leq \sqrt{n}} i \\
& =1+o(1)
\end{aligned}
$$

\section{REFERENCES}

1. R. C. Buck, Generalized asymptotic density, Amer. J. Math. 75(1953), 335-346. MR 14:854f

2. J. S. Connor, The statistical and strong p-Cesàro convergence of sequences, Analysis 8(1988), 47-63. MR 89k:40013

3. J. S. Connor and M. A. Swardson, Strong integral summability and the Stone-Čech compactification of the half-line, Pacific J. Math. 157 (1993), 201-224. MR 94f: 40007

4. J.S. Connor and M.A. Swardson, Measures and ideals of $C^{*}(X)$, Annals N.Y. Acad. Sci. 104 (1994), 80-91. MR 95b:54022

5. J.S. Connor and J. Kline, On statistical limit points and the consistency of statistical convergence, J. Math. Anal. Appl. 197 (1996), 392-399. MR 96m:40001

6. P. Erdös and G. Tenenbaum, Sur les densities de certaines suites d'entries, Proc. London Math. Soc. 59 (1989), 417-438. MR 90h:11087

7. H. Fast, Sur la convergence statistique, Colloq. Math. 2(1951), 241-244. MR 14:29c

8. J.A. Fridy, On statistical convergence, Analysis 5(1985), 301-313. MR 87b:40001

9. , Statistical limit points, Proc. Amer. Math. Soc. 118(1993), 1187-1192. MR 94e: 40008

10. J. Fridy and C. Orhan, Lacunary statistical summability, J. Math. Anal. Appl. 173 (1993), 497-504. MR 95f: 40004

11. J. Fridy and H.I. Miller, A matrix characterization of statistical convergence, Analysis 11 (1991), 59-66. MR 92e:40001

12. G.H. Hardy, Divergent Series, Oxford Univ. Press, London, 1949. MR 11:25a

13. K. Knopp, Zur Theorie der Limitierungsverfahren(Erste Mitteilung), Math. Zeit. 31(1930), 115-127.

14. I.J. Maddox, Steinhaus type theorems for summability matrices, Proc. Amer. Math. Soc. 45(1974), 209-213. MR 51:1192

15. _ Some analogues of Knopp's Core Theorem, Internat. J. Math. \& Math. Sci., 2(1979), 605,-614. MR 81m:40012

16. Statistical convergence in locally convex spaces, Math. Cambridge Phil. Soc. 104 (1988), 141-145. MR 89k:40012

17. H.I. Miller, A measure theoretical subsequence characterization of statistical convergence, Trans. Amer. Math. Soc. 347 (1995), 1811-1819. MR 95h:40010

18. I. Niven, H.S. Zuckerman, and H. Montgomery, An Introduction to the Theory of Numbers, Fifth Ed., Wiley, New York, 1991. MR 91i:11001

19. A. Zygmund, Trigonometric Series, 2nd Ed., Cambridge Univ. Press, 1979. MR 58:29731

Department of Mathematics and Computer Science, Kent State University, Kent, Оніо 44242-0001

E-mail address: fridy@mcs.kent.edu

Department of Mathematics, Faculty of Science, Ankara University, Ankara, 06100, TURKEY

E-mail address: orhan@science.ankara.edu.tr 